\title{
FENOLOGÍA DE DOS PLANTAS NUTRICIAS, Centrosema triquetrum Y Rorippa indica, PARA PRODUCCIÓN COMERCIAL DE MARIPOSAS EN LA RESERVA PAWAY, EN EL MUNICIPIO DE MOCOA (PUTUMAYO) ${ }^{1}$
}

\author{
Mildred Alejandra Ortiz-Martinez ${ }^{2}$ \\ Sandra Morales-Velasco ${ }^{3}$ \\ José Manuel Tobar-Mesa ${ }^{4}$
}

Recibido el 3 de diciembre de 2013, aprobado el 23 de noviembre de 2014 y actualizado el 28 de abril de 2015

\section{DOI: 10.17151/luaz.2015.41.7}

\section{RESUMEN}

El Instituto Alejandro von Humboldt registra aproximadamente 3800 especies de mariposas para Colombia y en particular 350 para la Amazonía; que por sus características se han posicionado como una alternativa de biocomercio, pero una limitante para la reproducción, es la selectividad en su alimentación (monófagas).

Por lo anterior, se realizó el estudio fenológico de dos plantas nutricias, Centrosema triquetrum y Rorippa indica, para la producción comercial de mariposas Morpho helenor, Ascia monuste y Leptophobia aripa, en la Reserva Paway (Mocoa Putumayo), para planificar la producción, garantizando calidad y cantidad.

Para la evaluación fenológica se tuvieron en cuenta cuatro fases (germinación, emergencia, desarrollo de las hojas y floraciónmadurez) y dos variables ambientales (temperatura y humedad relativa). El consumo de alimento se realizó suministrando forraje verde durante el desarrollo del ciclo larvario de las mariposas, en grupos de cinco orugas por especie. Paralelamente se calculó el porcentaje en materia seca para cada especie.

Se determinó que Rorippa indica presenta un ciclo fenológico de 74 días, Centrosema triquetrum al cabo de un año no había florecido. La humedad presentó una correlación altamente significativa $(p \leq 0,01)$ para el desarrollo de las dos especies vegetales y la temperatura $(p \leq 0,01)$ para $C$. triquetrum.

El ciclo larvario de Morpho helenor fue de 86 días, 20 para Ascia monuste y Leptophobia aripa, periodo en el que se les suministró forraje verde de la respectiva planta nutricia, consumiendo en promedio 22,9 gramos.

\section{PALABRAS CLAVE}

Plantas nutricias, ciclo larvario, variables ambientales. 


\title{
PHENOLOGY OF TWO NUTRITIOUS PLANTS, Centrosema triquetrum AND Rorippa indica, FOR COMMERCIAL \\ PRODUCTION OF BUTTERFLIES IN THE PAWAY NATURAL PARK , IN THE MUNICIPALITY OF MOCOA (PUTUMAYO)
}

\begin{abstract}
The Alexander von Humboldt Institute records about 3800 species of butterflies for Colombia and in particular 350 for the Amazon which, due to their characteristics, have been positioned as a biocommerce alternative though a limitation for reproduction is the selectivity in their diet (monophagous).
\end{abstract}

Therefore, the phenology study of two nutritious plants Centrosema triquetrum, and Rorippa indica was carried out, for the commercial production of Morpho helenor, Ascia monuste and Leptophobia aripa butterflies in the Paway natural park (Mocoa - Putumayo), to plan production, ensuring quality and quantity.

Four phases (germination, emergence, leaf development and flowering-maturity) and two environmental variables (temperature and humidity) were taken into account for the phenology assessment. Food intake was carried out supplying green fodder during larval development cycle of butterflies in groups of five caterpillars per species. Alongside the percentage of dry matter for each species was calculated.

It was determined that Rorippa indica presents a phenology cycle of 74 days, Centrosema triquetrum after a year had not bloomed. The humidity presented a highly significant correlation $(p \leq 0.01)$ for the development of the two vegetal species and temperature $(p \leq 0.01)$ for $C$. triquetrum .

The Morpho helenor larval cycle was 86 Days, 20 for Ascia monuste and Leptophobia aripa, period in which green forage of the respective nutritional plant was supplied, consuming on average 22.9 grams.

\section{KEY WORDS}

Nurtritious plants, larval cycle, environmental variables.

\section{INTRODUCCIÓN}

Las mariposas diurnas (Lepidóptera: Rhopalocera) cumplen funciones importantes en los ecosistemas: contribuyen a la polinización de las flores, a la alimentación de otros animales y en general a la renovación de la vida silvestre (Constantino, 1996). En la actualidad se han posicionado como una alternativa económica de exportación, proyectada en seis sectores: coleccionistas, artesanías e industrias de adornos, museos, compra de escamas para la fabricación de chips, granjas o 
vivarios de mariposas y como mascotas. Los cuatro primeros sectores requieren mariposas disecadas y preservadas, mientras que los dos últimos requieren los individuos vivos, en forma de orugas, pupas recién formadas y adultas (Instituto Alexander von Humboldt, 2003; Gómez y Lozada, 2005).

Muchas especies son monófagas, es decir, que sus orugas se alimentan solamente de una planta en particular, por lo que si esta desaparece, lo mismo ocurrirá con la mariposa (Aguilar, 2004). Por eso, es importante conocer el ciclo fenológico y la caracterización morfológica de las especies nutricias de las mariposas, de tal manera que permita promover su cultivo con fines de producción de follaje para la alimentación de distintas orugas; brindando una nueva alternativa productiva que permite la conservación de varias especies de la biodiversidad y la sustentabilidad económica a pequeños productores (Gómez, 2010).

La presente investigación tuvo como objetivo estudiar la morfología y fenología de las plantas Centrosema triquetrum y Rorippa indica, para la producción comercial de mariposas en la Reserva Paway, en el municipio de Mocoa (Putumayo), garantizando la producción forraje verde.

\section{MATERIALES Y MÉTODO}

\section{Localización}

La investigación se realizó en la Reserva natural Paway ubicada en el municipio de Mocoa, vereda San José del Pepino, entre las coordenadas $1^{\circ} 05^{\prime} 14,9^{\prime \prime} \mathrm{N}$ y $76^{\circ} 37^{\prime} 58,9^{\prime \prime} \mathrm{W}$, altitud dese los 310 hasta los $670 \mathrm{~m}$, temperatura media de $23,5^{\circ} \mathrm{C}$, pluviosidad de $4708 \mathrm{~mm}$ anuales y una humedad relativa de $85 \%$.

Esta reserva natural cuenta con un área de 12,7 ha. La vegetación es típica de un bosque húmedo tropical, constituido por un bosque de galería de aproximadamente 4 ha, bosque de 6 ha y un área intervenida de más de 2 ha (Figura 1). 


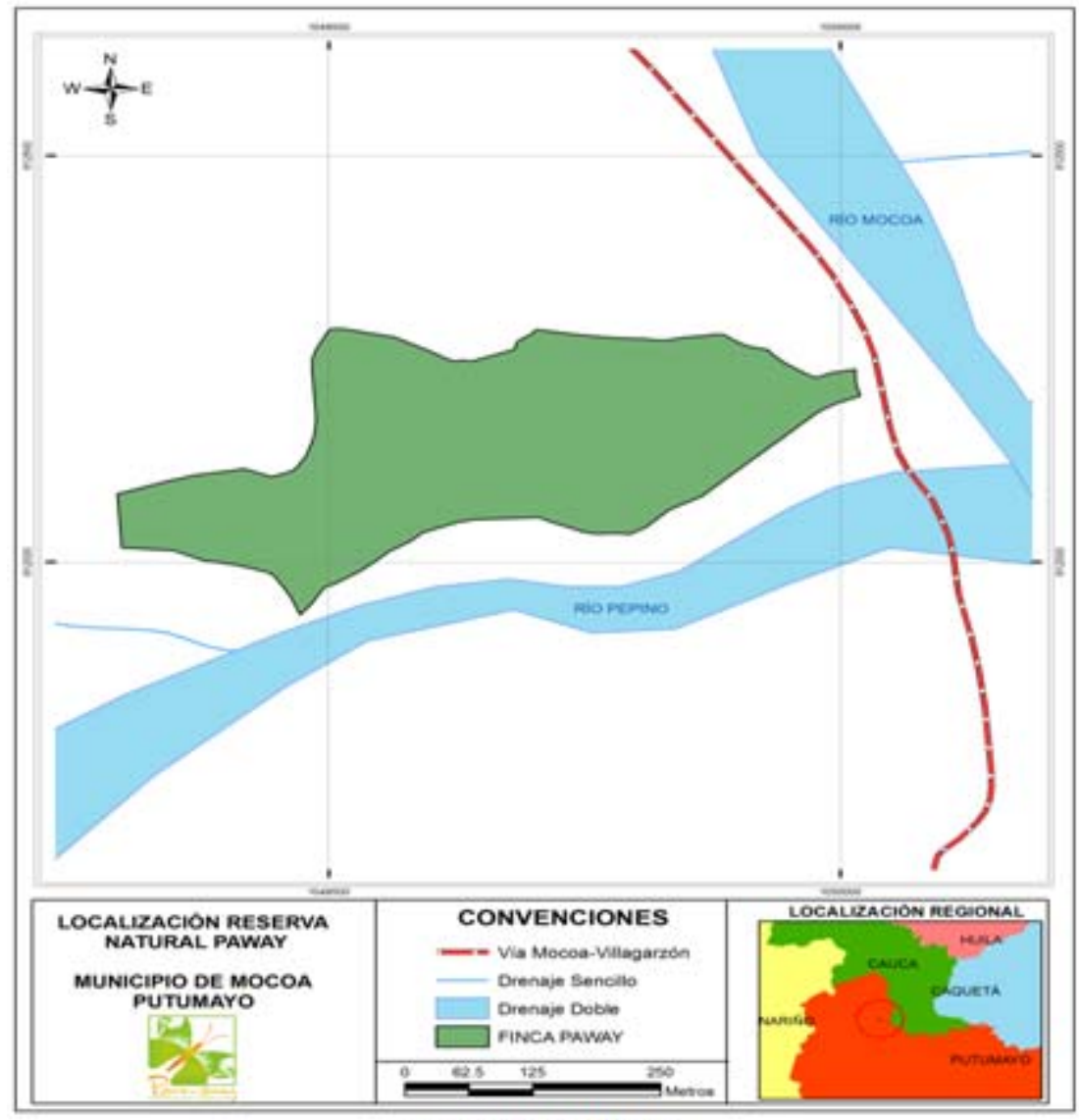

Fuente: los autores.

Figura 1. Mapa de localización del área de estudio.

\section{Fenología}

Se recolectaron semillas en la Reserva Paway; para el caso de $R$. indica al borde de carretera y el C. triquetrum en el sotobosque.

Las semillas se sembraron en eras de $1 \times 3 \mathrm{~m}$, el $R$. indica se sembró a una distancia de $5 \mathrm{~cm}$ entre plantas y $10 \mathrm{~cm}$ entre surcos, a $1 \mathrm{~cm}$ de profundidad, para un total de 600 semillas. Las semillas de $C$. triquetrum fueron 75 , a una distancia de $20 \mathrm{~cm}$ entre plantas y $40 \mathrm{~cm}$ entre surcos, a $4 \mathrm{~cm}$ de profundidad (Figura 2). 


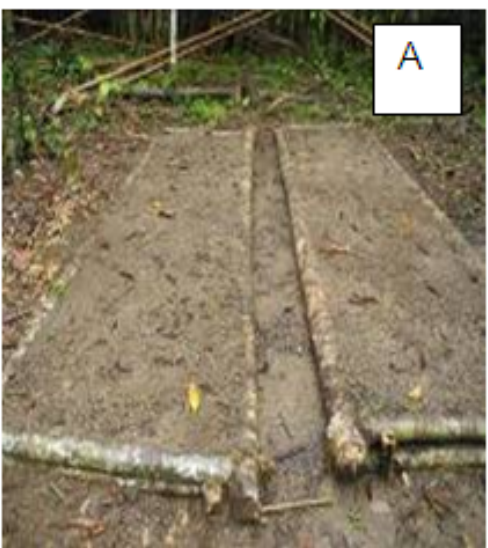

A. Eras

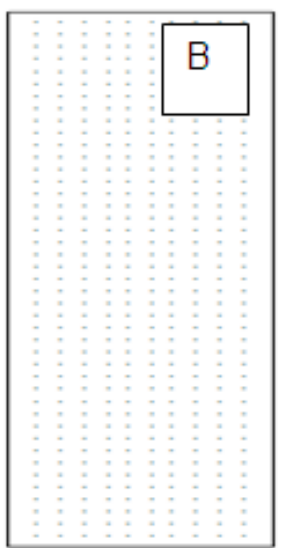

B. Siembra $R$. indica

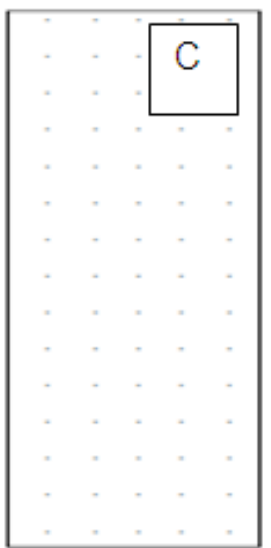

C. Siembra C. triquetrum

Fuente: los autores.

Figura 2. Reproducción de las semillas en la Reserva Paway.

Los registros se realizaron cada 8 días a partir de la siembra y durante el tiempo de desarrollo de las plantas, hasta que finalizó el tiempo del estudio (Ruiz, 1993).

Se efectuaron observaciones fenológicas para las fases de germinación, desarrollo de las hojas, floración, fruto y maduración completa (De la Morena et al., 1986; Monasterio et al., 2007); los datos se midieron hasta cuando el $50 \%$ de las semillas alcanzaron el desarrollo en cada una de las fases. Las variables evaluadas fueron:

Hoja. El tamaño se midió en centímetros $(\mathrm{cm})$ y, solo se tomó el largo y ancho. El número de hojas verdaderas se evalúo realizando un conteo de las hojas, que alcanzaron una longitud mínima de $4 \mathrm{~cm}$ por planta.

Tallo. La altura se midió en centímetros $(\mathrm{cm})$ y, se tomó desde el suelo hasta la inserción de la última hoja formada; el diámetro promedio se tomó en centímetros $(\mathrm{cm})$ y, se midió de la máxima extensión de un lado a la máxima extensión del lado opuesto. Para C. triquetrum se evaluó el número de lados y el hábito de crecimiento.

Inflorescencia. Longitud, número de plantas florecidas, número de flores por inflorescencia y coloración de la flor.

Fruto. Longitud, ancho, número de semillas por fruto y coloración.

Semilla. Cantidad de semillas que produce cada fruto.

Para la evaluación fue necesario tener en cuenta los factores climáticos (humedad y temperatura), que fueron tomados tres veces por semana en el sitio del ensayo. Los datos colectados se organizaron en una matriz en Excel para una correlación de 
Pearson entre los datos, las variables ambientales y el desarrollo vegetativo de las plantas, utilizando el programa SPSS 15.0.

\section{Producción de forraje para alimentación de orugas}

Para la producción de alimento, se determinó el porcentaje de materia seca, seleccionando una planta tipo de cada especie, cortando el forraje verde proveniente de las hojas (100 g para $R$. indica y $250 \mathrm{~g}$ para $C$. triquetrum) y secadas a una temperatura de $115^{\circ} \mathrm{C}$.

Para el consumo de forraje de Morpho helenor, se seleccionaron orugas de cinco $\mathrm{mm}$ (un día de eclosión), colocadas en la mañana sobre una hoja previamente pesada (tamaño promedio de $13 \times 9 \mathrm{~cm}$ ) y retirada en la tarde para calcular la cantidad de alimento ingerido (Figura 3 ).

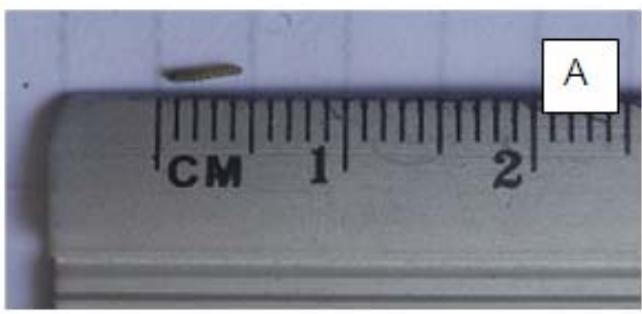

A. Orugas de $3 \mathrm{~mm}$.

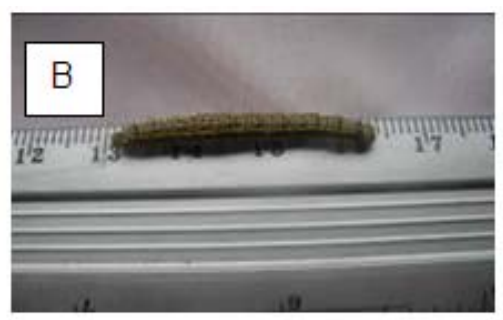

B. Orugas de $3 \mathrm{~cm}$.

Fuente: los autores.

Figura 3. Orugas de mariposas para el consumo de alimento.

Una vez las orugas alcanzaron un tamaño de tres $\mathrm{cm}$ se trasladaron a un habitáculo, sobre una planta previamente sembrada en materas, garantizando alimento fresco (Figura 4).

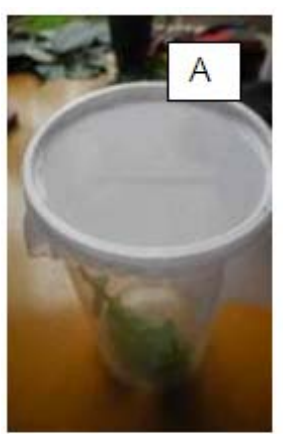

A. Tarrinas con orugas

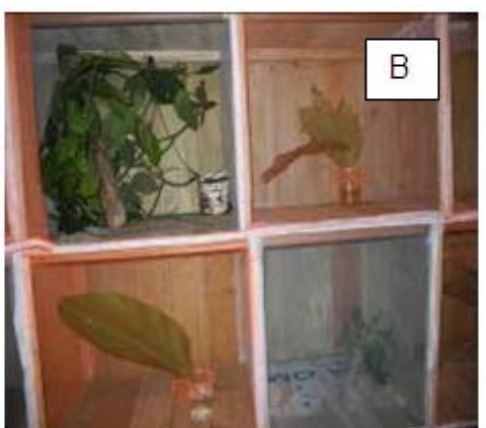

B. Habitáculos.

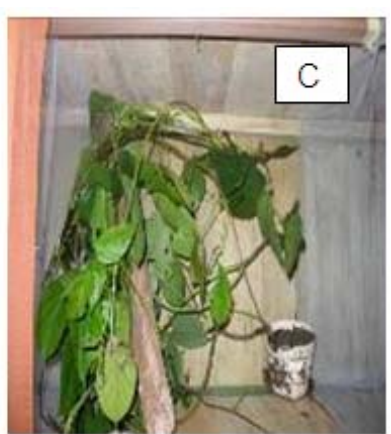

C. Orugas alimentándose en habitáculo.

Fuente: los autores

Figura 4. Orugas alimentándose en tarrinas y habitáculos.

La planta se cambió cada cinco días, pesándose para valorar el consumo de forraje, actividad repetitiva hasta el estadio de prepupa que es cuando dejan de alimentarse. 


\section{RESULTADOS Y DISCUSIÓN}

\section{Fenología de Centrosema triquetrum}

La fase de germinación se presentó a los 29 días, la primera expresión fue la aparición de la radícula, desarrollándose posteriormente entre dos y cinco raíces secundarias (Tabla 1).

Tabla 1. Fenología de Centrosema triquetrum

\begin{tabular}{|l|c|c|c|c|c|c|c|c|c|c|}
\hline Fase & Germinación & \multicolumn{3}{|c|}{$\begin{array}{c}\text { Emergencia y } \\
\text { desarrollo de hojas }\end{array}$} & Floración & \multicolumn{5}{|c|}{ Maduración } \\
\hline Subfase & & Inicio & $50 \%$ & $75 \%$ & & IFF & FCD & IFS & SCD & MC \\
\hline Días & 29 & 41 & 74 & 229 & & & & & & \\
\hline
\end{tabular}

IF: Inicio de formación de frutos. FCD: Frutos completamente desarrollados. IFS: Inicio de formación de semillas. SCD: Semillas completamente desarrolladas. MC: Madurez completa.

El comienzo de la emergencia se dio a los 41 días, se observó el crecimiento del epicótilo entre los cotiledones y el primer par de hojas (Figura 5).

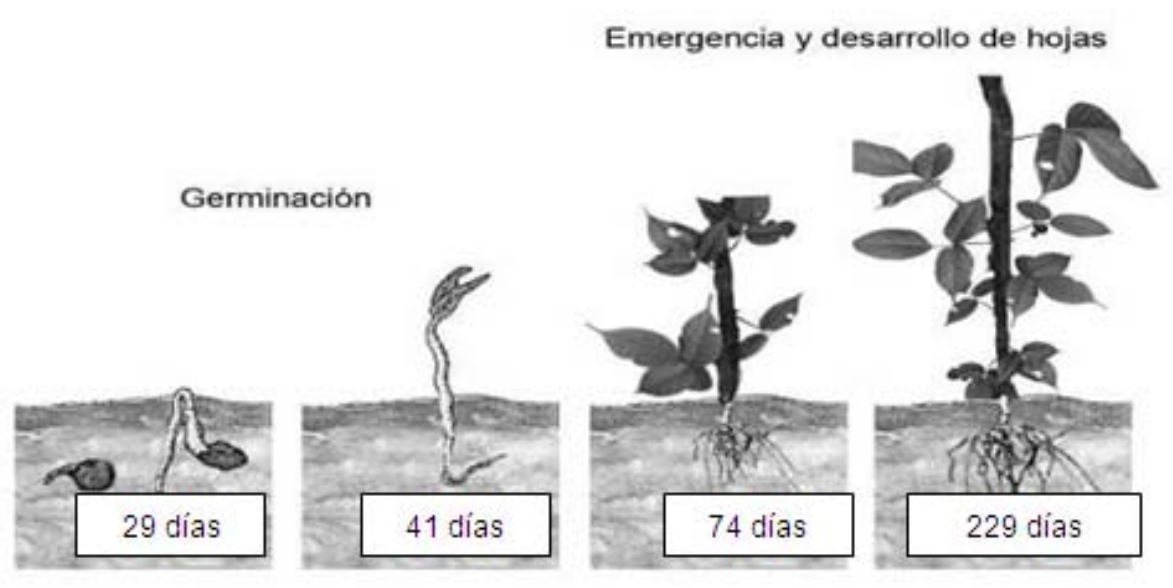

Fuente: Ios autores.

Figura 5. Desarrollo fenológico de Centrosema triquetrum.

A los 74 días comenzó el despliegue de las hojas primarias, iniciando con la formación del tallo y de las hojas trifoliadas. A los 229 días, el $75 \%$ de las plantas se encontraron en esta fase (Figura 6). 


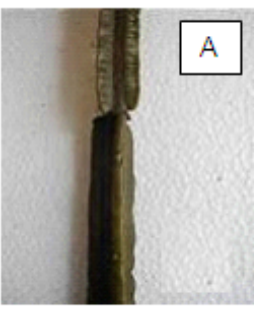

A) Flor.

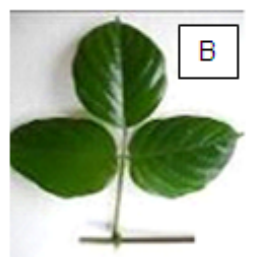

B) Hojas trifoliadas.

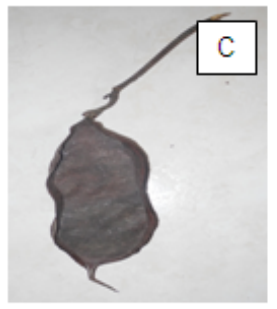

C) Fruto

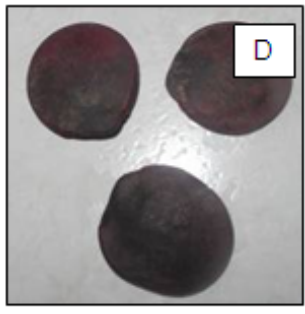

D) Semilla

Fuente: los autores

Figura 6. Estructuras morfológicas de Centrosema triquetrum.

La forma del tallo es angular, presenta ramificaciones de crecimiento monopodial, de consistencia herbácea, de color verde oscuro con manchas cafés.

Los análisis realizados muestran una correspondencia entre las variables ambientales y el crecimiento de la planta Centrosema triquetrum, que pudo influenciar en la fenología de la especie y en particular la floración, que no se presentó durante la evaluación. La temperatura tuvo una correlación altamente significativa $(p \leq 0,01)$ para el largo de hoja $(r=0,59)$, ancho de hoja $(r=0,40)$ y número de hojas $(r=0,53)$, mostrando que a mayor temperatura hay incremento en las hojas.

El mayor desarrollo de las hojas se dio entre los días 140 y 260 , tiempo durante el cual en promedio se tuvo una temperatura de $28,58^{\circ} \mathrm{C}$ (Figura 7 ).

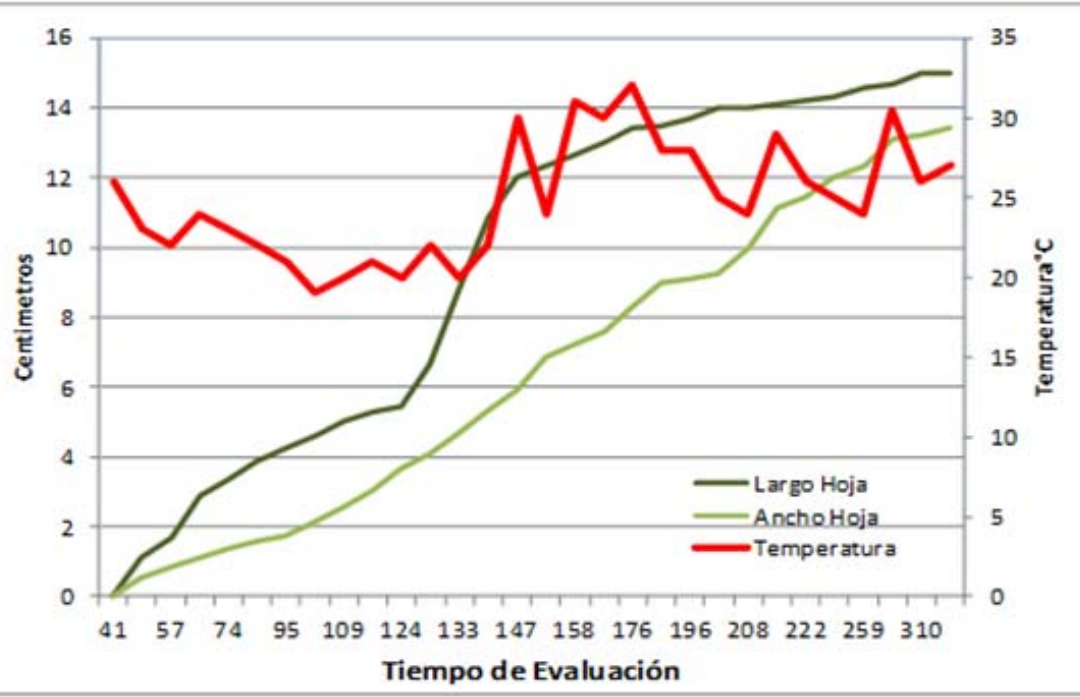

Figura 7. Relación variables de la hoja de Centrosema triquetrum y temperatura.

Esto posiblemente se debe a que algunas especies de leguminosas, reaccionan mejor a un valor óptimo de temperatura (30 y $35^{\circ} \mathrm{C}$ ) para el desarrollo de sus funciones, con una alta sensibilidad a las bajas temperaturas, cuyos efectos negativos durante el periodo de crecimiento pueden afectar las tasas de 
asimilación y traslocación de metabolitos (Kantolic, 2004), llegando incluso a provocar daños físicos en el aparato fotosintético lo cual limita el crecimiento (Allen y Ort, 2001), porque influencia los procesos bioquímicos fisiológicos básicos (síntesis, transporte y degradación de sustancias en las plantas), ya que poseen relación con el mantenimiento de la integridad de las membranas (Rodríguez, 2004; Satisha et al., 2007).

Para la variable humedad relativa, presentó correlaciones negativas con una significancia de $p \leq 0,01$, para el largo de hojas $(r=-0,81)$, ancho de hoja $(r=-0,74)$, hojas por planta $(r=-$ $0,73)$, altura del tallo $(r=-0,77)$ y diámetro tallo $(r=-0,69)$, mostrando que a mayor humedad se presenta un menor desarrollo de la planta (Figura 8).

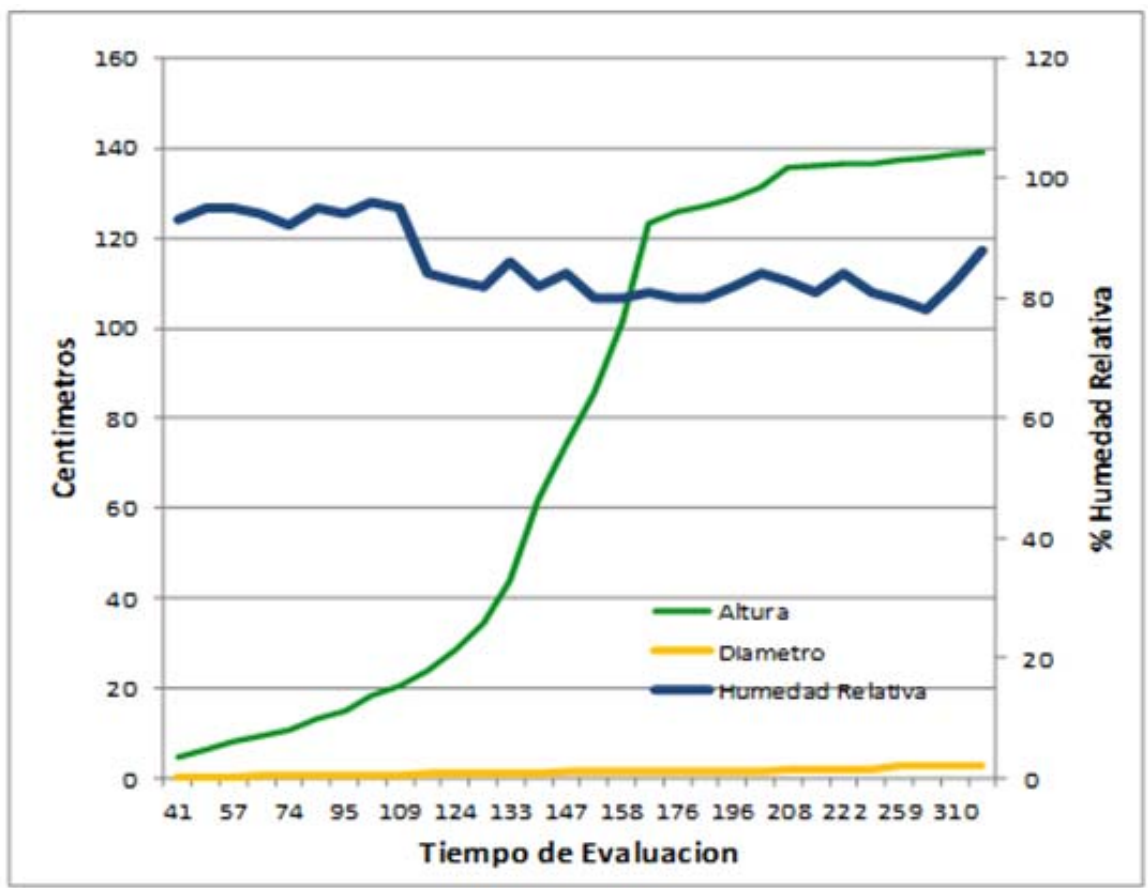

Figura 8. Relación variables del tallo de Centrosema triquetrum y humedad relativa.

Se puede inferir que el alto porcentaje de lluvias influyó sobre la humedad del suelo, observándose que a partir del día 150, cuando el porcentaje de humedad relativa disminuye, se incrementa el crecimiento del tallo; debido a que el exceso de humedad del suelo puede provocar estrés en los cultivos (Kantolic, 2004), generalmente en los suelos mal drenados y donde las precipitaciones son altas durante todo el año (4708 $\mathrm{mm} / \mathrm{año}$ ), pueden provocar anoxia en las raíces, afectando su respiración aeróbica, absorción de minerales y agua (Baruch, 1994).

\section{Fenología de Rorippa indica}

Se observaron las cuatro fases fenológicas planteadas al inicio del estudio: germinación, emergencia y desarrollo de las hojas, floración y maduración. La duración de todas las fases fue de 74 
días. La germinación se presentó al sexto día después de la siembra, donde la emergencia y desarrollo de las hojas, inició con la elongación entre los cotiledones y la radícula (Tabla 2).

Tabla 2. Fenología de Rorippa indica

\begin{tabular}{|l|c|c|c|r|r|r|r|r|}
\hline \multicolumn{1}{|c|}{ Fase } & Germinación & $\begin{array}{r}\text { Emergencia y desarrollo } \\
\text { de hojas }\end{array}$ & Floración & \multicolumn{4}{|c|}{ Maduración } \\
\hline Subfase & & & & IFF & FCD & IFS & SCD & MC \\
\hline Días & 6 & 21 & 39 & 48 & 53 & 60 & 71 & 74 \\
\hline
\end{tabular}

IF: Inicio de formación de frutos. FCD: Frutos completamente desarrollados. IFS: Inicio de formación de semillas. SCD: Semillas completamente desarrolladas. MC: Madurez completa.

Los días de floración fueron de 39 (flores pequeñas y amarillas), 48 para la madurez con la formación de frutos y de 60 para las semillas (Figura 9).

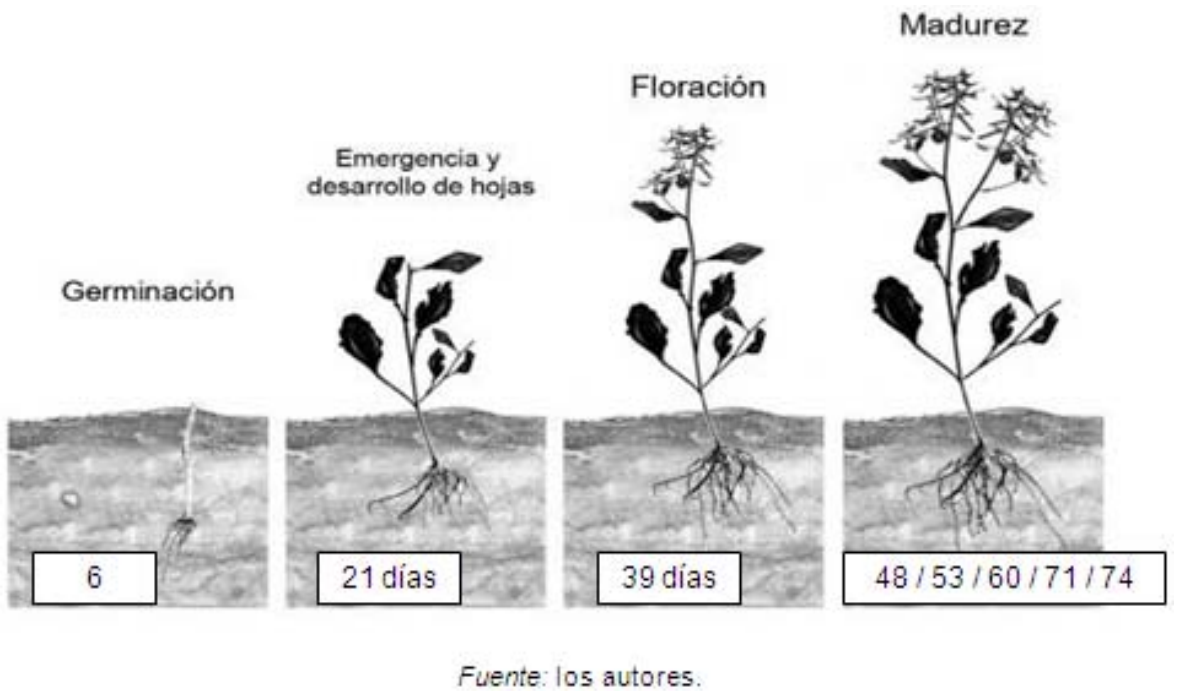

Figura 9. Desarrollo fenológico de Rorippe indice.

La floración inició con la aparición de las primeras inflorescencias, el número por planta fue de 7 , con 5 flores por cada una de ellas (Figura 10).

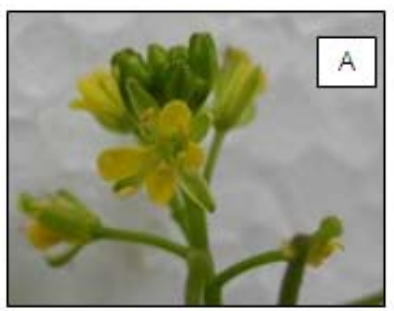

A) Flor.

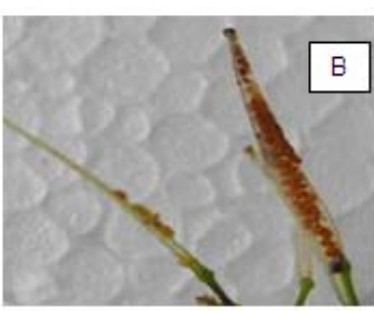

B) Fruto

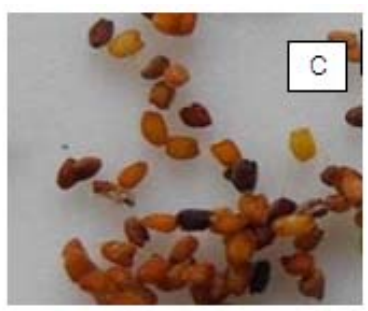

C) Semilla.

Fuente: los autores.

Figura 10. Estructuras morfológicas de Rorippa indica. 
Una vez las flores fueron fecundadas, se inició la formación de frutos, con un promedio de 20 por planta, de $2 \mathrm{~cm}$ de largo, color verde claro y al final de la etapa, tomaron un color café. El número de semilla por fruto fue de 59 .

Para las variables ambientales, no se encontró correlación entre la temperatura y las variables de la planta Rorippa indica. La humedad relativa presentó significancia alta pero negativa $(p \leq$ $0,01)$, evidenciando que a menor humedad mayor crecimiento de la planta, para el largo de hoja $(r=-0,773)$, ancho de hoja $(r=-$ $0,834)$, número de hojas $(r=-0,650)$, altura de la planta $(r=-$ $0,929)$, diámetro $(r=-0,775)$, número de inflorescencias por planta $(r=-0,692)$, flores por inflorescencia $(r=-0,682)$, frutos $(r$ $=-0,95)$ y semillas $(r=-0,265)$ (Figura 11).

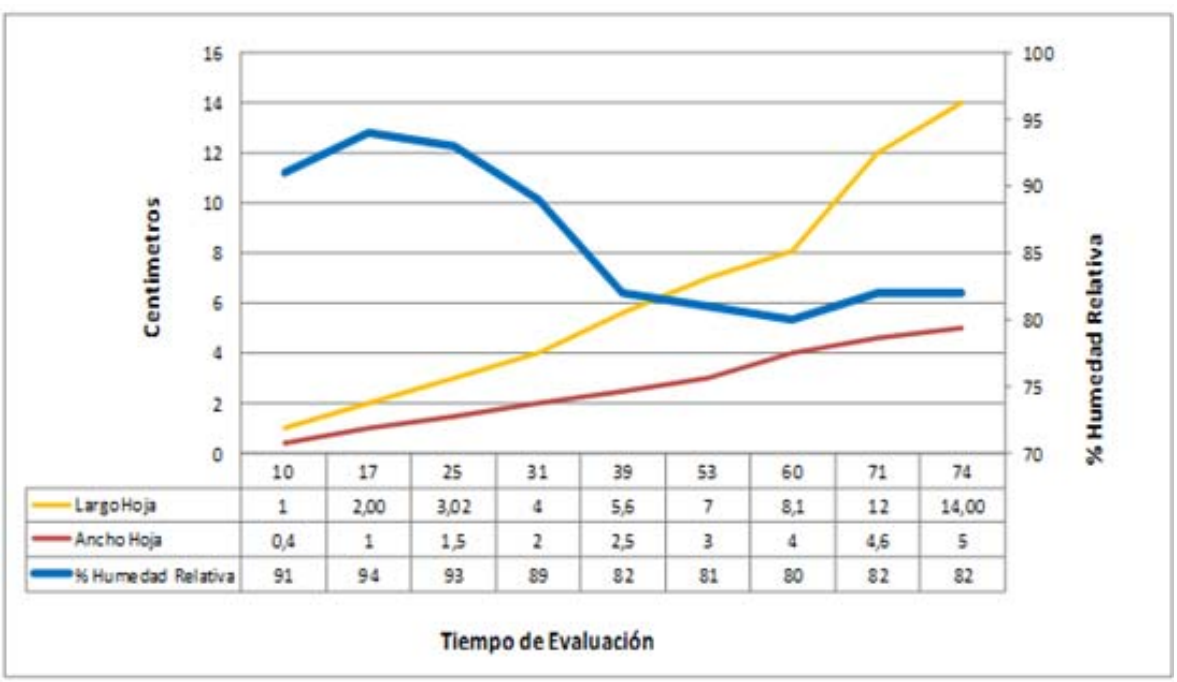

Figura 11. Relación de las variables hojas de Rorippa indica y humedad relativa.

Al disminuir la humedad se incrementó el desarrollo de las hojas y alargamiento de la planta. El crecimiento del tallo, se dio de forma rápida a partir del día 21 , a medida que se reducía el porcentaje de humedad, alcanzando un diámetro promedio de 31,8 cm (Hernández, 2008; Olivares, 2008) (Figura 12).

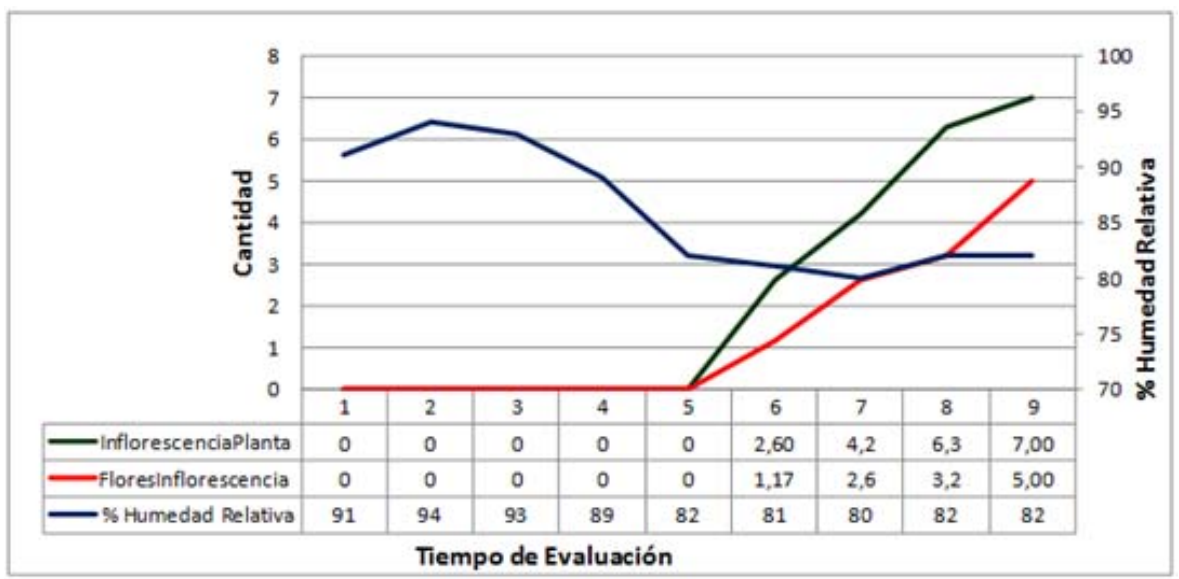

Figura 12. Relación variable de floración de Rorippa indica y humedad relativa. 
La humedad relativa, se mantuvo entre 81 y $82 \%$ durante la floración hasta el inicio de la formación del fruto. Durante la época de siembra se presentaron episodios de lluvia intensos, situación que ocasionó el anegamiento del suelo, produciendo saturamiento en los macroporos del suelo y asfixia celular a nivel radicular, perdiendo funcionalidad sus raíces y provocando marchitamientos de las plantas (Monasterio et al., 2007).

\section{Producción de forraje para alimentación de orugas}

En la Tabla 3 se registra la producción de alimento, con base en el porcentaje en materia seca del forraje verde generado por las plantas nutricias.

Tabla 3. Producción en materia seca

\begin{tabular}{|l|c|c|c|}
\hline Planta nutricia & Forraje verde $(\mathrm{g})$ & Peso seco $(\mathrm{g})$ & Materia seca $(\%)$ \\
\hline Centrosema triquetrum & 250 & 72 & 28,8 \\
\hline Rorippa indica & 100 & 17,23 & 17,23 \\
\hline
\end{tabular}

El suministró de forraje verde fue acorde al desarrollo larvario de cada mariposa; de 86 días para Morpho helenor y de 20 para Ascia monuste y Leptophobia aripa.

En promedio las cinco orugas de Morpho helenor consumieron $22,9 \mathrm{~g}$ y Ascia monuste y Leptophobia aripa $6,8 \mathrm{~g}$ de hojas frescas por grupo (Figura 13).

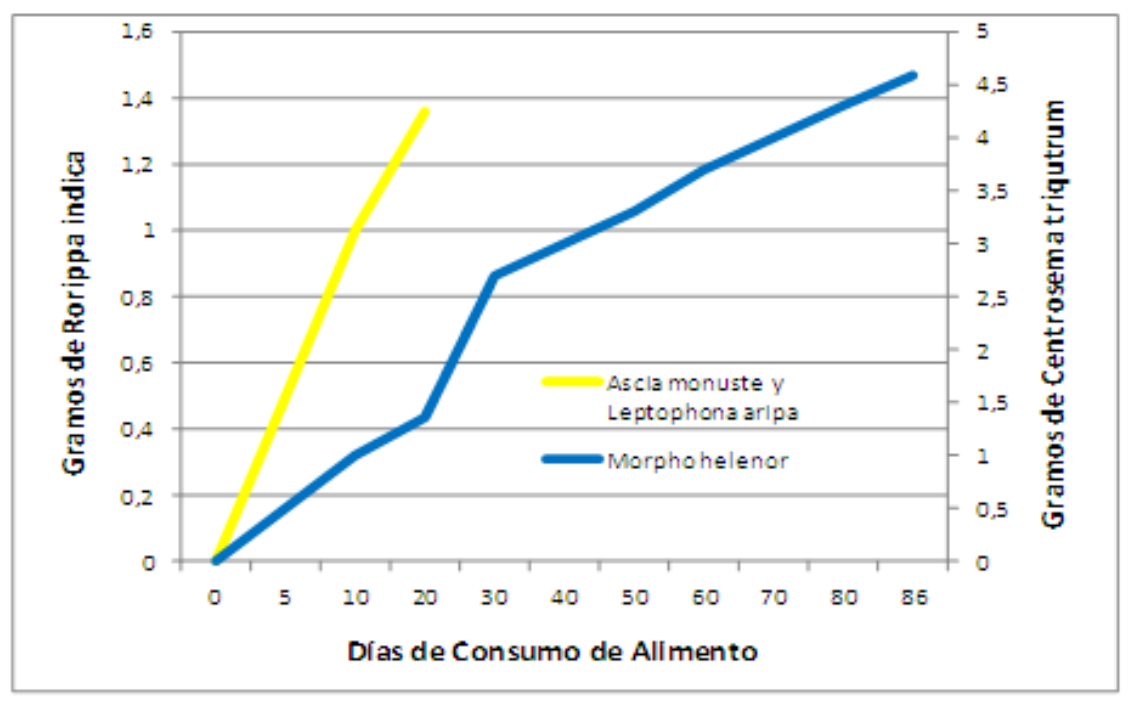

Figura 13. Consumo de alimento por oruga de Morpho helenor, Ascia monuste y Leptophobia aripa, en forraje verde.

Es de anotar el incremento del consumo, el cual está dado por el desarrollo de la larva, que puede alcanzar hasta $8 \mathrm{~cm}$ para Morpho helenor y $4 \mathrm{~cm}$ para las otras especies. 
En la Tabla 4 se anotan los cálculos de consumo por planta y por especie.

Tabla 4. Consumo de alimento en materia seca

\begin{tabular}{|c|c|c|c|c|c|}
\hline $\begin{array}{c}\text { Especie de } \\
\text { mariposa }\end{array}$ & Planta nutricia & Hojas/Planta & $\begin{array}{c}\text { Peso } \\
\text { hoja }\end{array}$ & $\begin{array}{c}\text { Consumo } \\
\text { forraje } \\
\text { verde/oruga }\end{array}$ & $\begin{array}{c}\text { Consumo } \\
\text { materia } \\
\text { seca/oruga }\end{array}$ \\
\hline Morpho helenor & $\begin{array}{c}\text { Centrosema } \\
\text { triquetrum }\end{array}$ & 107 & $2,3 \mathrm{~g}$ & $4,58 \mathrm{~g}$ & $1,32 \mathrm{~g}$ \\
\cline { 1 - 1 } Ascia monuste & Rorippa indica & 14 & $0,8 \mathrm{~g}$ & $1,36 \mathrm{~g}$ & $0,23 \mathrm{~g}$ \\
\hline $\begin{array}{c}\text { Leptophobia } \\
\text { aripa }\end{array}$ & Rorippa indica & 14 & & \\
\hline
\end{tabular}

Partiendo de una producción de 500 mariposas Morpho helenor, por ciclo bilógico de la especie, se deben suministrar $2290 \mathrm{~g}$ en forraje verde de Centrosema triquetrum.

Para Ascia monuste y Leptophobia aripa, se deberán producir $680 \mathrm{~g}$ de Rorippa indica; para cada una de las especies, lo que indica que sería necesario mantener al menos 10 plantas, por ciclo para cada especie.

\section{CONCLUSIONES Y RECOMENDACIONES}

La humedad relativa afectó el desarrollo de las dos especies, limitando el crecimiento vegetativo de las plantas y en particular la floración del Centrosema triquetrum.

Se deben sembrar 10 plantas de $C$. triquetrum cada 41 días y de $R$. indica 61 plantas cada 21 días para mantener una población de 500 mariposas por especie, dada la fase de emergencia y desarrollo de hojas; y con el fin de mantener ciclos constantes de producción de mariposas.

Se requiere seguir investigando sobre las dos plantas en aspectos como la calidad nutricional y realizar estudios similares en otras regiones del país, para conocer el comportamiento en regiones más secas.

Estudiar la respuesta de las plantas nutricias a un manejo agronómico como fertilización, densidad de siembra y asociación con especies arbóreas.

Realizar evaluaciones de las plantas frente a otras variables meteorológicas, como brillo solar y precipitación. 


\section{AGRADECIMIENTOS}

Los autores agradecen a la Universidad del Cauca, y al profesor Bernardo Ramírez, Director del Herbario de la Universidad del Cauca, el cual contribuyó con la clasificación de las plantas, y a PAWAY, Centro Ecoturístico Amazónico SAS.

\section{POTENCIAL CONFLICTO DE INTERESES}

No hay conflicto de intereses.

\section{FUENTES DE FINANCIACIÓN}

No se tuvo.

\section{REFERENCIAS}

- Aguilar, Z. (2004). Flowering on community level in a terra firme forest in ecuadorian amazon. Lyiona, 7, 116-123.

- Allen, D.J. y Ort, D.R. (2001). Impacts of chilling temperatures on photosynthesis in warm-climate plants. Trends in Plant Science, 6, 36-42.

- Baruch, Z. (1994). Las respuestas a la sequía y las inundaciones en las gramíneas forrajeras tropicales. I. - La asignación de biomasa, crecimiento de las hojas y los nutrientes minerales. Plant \& Soil, 164, 87-96.

- Constantino, L.M. (1996). Ciclos de vida y plantas hospederas de lepidópteros con potencial económico en condiciones de colinas bajas del Chocó biogeográfico. En Memorias. II Seminario. Investigación y Manejo de Fauna para la Construcción de Sistemas Sostenibles. INCIVA, U. Javeriana, IMCA, CIPAV, WWF, Instituto von Humboldt. Cali, marzo 28-30.

- De la Morena, I., Ramos, J.M. y García del Moral, L.F. (1986). Análisis del crecimiento y de la producción de grano en cultivos de cebada bajo las condiciones ambientales de la provincia de Granada. II. Evolución y supervivencia de los tallos hijos. Anales de Edafología y Agrobiología, 779-796.

- Gómez, J.A. y Lozada, P.A. (2005). Análisis del desarrollo empresarial de 100 iniciativas de biocomercio sostenible en Colombia. Bogotá D.C: Instituto de Investigación de Recursos Biológicos Alexander von Humboldt.

- Gómez, M.d.R. (2010). ¿Criando Mariposas o Enfermedades? Proyectos de conservación y desarrollo con comunidades indígenas en la Amazonia Colombiana. Bogotá: Uniandes.

- Hernández, M.I. (2008). Evaluación agronómica de fertilizantes líquidos cubanos en el cultivo protegido del tomate (Solanum lycopersicum L.) Híbrido HA 3019. Cultivos Tropicales, 29(1), 7382. 
- Instituto de Investigación de Recursos Biológicos Alexander von Humboldt. (2003). Plan de aprovechamiento y uso de los recursos naturales: Guía para empresas de biocomercio. Bogotá: IAVH.

- Kantolic, A. (2004). Elementos centrales de ecofisiología del cultivo de soja. En Díaz Zorita, M. y Duarte, G. (Eds.), Manual práctico para la producción de soja. 1ra edición. Buenos Aires: Editorial Hemisferio Sur.

- Monasterio, P., García, P., Alejos, G., Pérez, A., Tablante, J., Maturet, W. y Rodríguez, L. (2007). Propuesta para la evaluación fenológica del cultivo de maíz en Venezuela. Revista Ciencia y Producción Vegetal, 59-68.

- Olivares, J.P. (2008). Fijación Biológica de Nitrógeno (Última actualización: 08/02/2008), Paper, Estación Experimental del Zaidín, SCIC, Granada, España.

- Rodríguez, A. (2004). Estudios preliminares sobre la influencia de diferentes factores ambientales en la productividad del Tagasaste (Chamaecytisus proliferus ssp.). Reunión Científica de la Sociedad Española para el Estudio de los Pastos. Salamanca.

- Ruiz, A. (1993). Caracterización Fenológica del Guayabo (Psidium guayava L.). Tesis de Maestría en Ciencias. Colegio de Postgraduados, Montecillo, México.

- Satisha J., Prakash, G.S., Bhatt, R.M. y Sampath Kumar, P. (2007). Physiological mechanisms of water use efficiency in grape rootstocks under drought conditions. International journal of Agricultural Research, 2, 159-164.

1. Artículo de investigación realizado en la reserva ecológica Paway, inscrito en el Grupo de Investigación Nutrición Agropecuaria de la Facultad de Ciencias Agrarias de la Universidad del Cauca.

2. Universidad del Cauca. Facultad de Ciencias Agrarias. Ingeniera Agropecuaria.

3. Universidad del Cauca. Facultad de Ciencias Agrarias. Departamento de Ciencias Agropecuarias. Grupo de Investigación Nutrición Agropecuaria. Ecóloga M.Sc.

4. Universidad del Cauca. Facultad de Ciencias Agrarias. Departamento de Ciencias Agropecuarias. Grupo de Investigación SISIMPRO. Ingeniero Agrónomo. M.Sc.

Para citar este artículo: Ortiz-Martinez M. A., Morales-Velasco s., Tobar-Mesa J. M. (2015). Fenología de dos plantas nutricias, Centrosema triquetrum y Rorippa indica, para producción comercial de mariposas en la Reserva Paway, en el municipio de Mocoa (Putumayo). Revista Luna Azul, 41, 116-130. Recuperado de

http://lunazul.ucaldas.edu.co/index.php?option=content\&tas $k=$ view\&id=1058 